\title{
Different orientation patterns of cellulose nanocrystal films prepared from aqueous suspensions by shearing under evaporation
}

\author{
$\operatorname{AUTHOR}(\mathrm{S})$ : \\ Tatsumi, Mio; Teramoto, Yoshikuni; Nishio, \\ Yoshiyuki
}

\section{CITATION:}

Tatsumi, Mio ...[et al]. Different orientation patterns of cellulose nanocrystal films prepared from aqueous suspensions by shearing under evaporation. Cellulose 2015, 22(5): 2983-2992

ISSUE DATE:

2015-10

URL:

http://hdl.handle.net/2433/202568

\section{RIGHT:}

The final publication is available at Springer via http://dx.doi.org/10.1007/s10570-015-0722-z.; The full-text file will be made open to the public on 07 August 2016 in accordance with publisher's 'Terms and Conditions for Self-Archiving'; This is not the published version. Please cite only the published version.; この論文は出版社版でありません。引用の際 には出版社版をご確認ご利用ください。 
1 Different orientation patterns of cellulose nanocrystal films prepared from aqueous

2 suspensions by shearing under evaporation

3

4

5 Mio Tatsumi, Yoshikuni Teramoto, Yoshiyuki Nishio*

6

7 Division of Forest and Biomaterials Science, Graduate School of Agriculture, Kyoto 8 University, Sakyo-ku, Kyoto 606-8502, Japan

9

$10 \quad{ }^{*}$ To whom correspondence should be addressed.

11 E-mail: ynishio@kais.kyoto-u.ac.jp (Y. Nishio). Tel.: +81 75753 6250. Fax: +81 75753 126300. 
Abstract: Oriented films were prepared from aqueous suspensions of cellulose nanocrystal (CNC; microfibril fragment of sulfuric acid-hydrolyzed cotton) by a shearing method. Rotating glass vials each containing a 3-4 wt \% CNC/water suspension under evaporation resulted in formation of translucent films of CNC per se. Structural characterization of the dry films was carried out by use of X-ray diffractometry and optical and scanning electron microscopy. The orientation pattern of CNCs in the films was much affected by $\mathrm{pH}$ condition of the starting suspensions; that is, the longitudinal axes of CNCs aligned preferentially perpendicular to the shear direction (SD) in the acidic condition of $\mathrm{pH}=2.0$, while an ordinary orientation of CNCs aligning parallel to SD was observed in the neutral condition of $\mathrm{pH}=6.7$ (adjusted with $\mathrm{NaOH}$ addition to the acidic suspension, however). To interpret the two distinct orientation patterns, first, it was inspected whether a mesomorphic ordered phase arrived or not in the two sheared and dried suspensions, different from each other in the counterions of surface-sulfated CNCs. As to the orientation development from the suspension of $\mathrm{pH}=2$, it was particularly assumed that the arising nematic planar domains would have been rolled up into a transversely extended body with the director perpendicular to SD. For the two film preparations, the orientation parameter of the longitudinal axis of CNC was quantified by WAXD intensity measurements, and the data were compared with those for other CNC-oriented materials such as CNC/polymer composites synthesized by immobilizing CNC suspensions via magnetic field application.

Keywords: Cellulose nanocrystal; Orientation; Solid film; Aqueous suspension; Shear flow 


\section{Introduction}

Fragmented microfibrils of cellulose, commonly termed cellulose nanocrystals (CNCs), are highly crystalline rod-like particles obtained by acid hydrolysis of native cellulose fibers (Marchessault et al. 1959; Revol et al. 1992). Particularly, CNCs prepared by the hydrolysis with sulfuric acid show an adequate dispersibility in water due to the negative charge of their sulfated surface. The resulting colloidal CNC suspension manifests a unique character to form a self-ordering mesoscopic structure via spontaneous phase separation (Revol et al. 1992; Gray 1994; Dong et al. 1996; Dong at al. 1998); that is, above a critical concentration of CNC (typically 3-5 wt \% for cotton-derived CNCs), the visually homogeneous suspension separates into an upper random phase (isotropic) and a lower ordered phase (anisotropic) in the course of quiescent standing. In the anisotropic phase, CNCs as mesogen form a chiral nematic organization.

In recent years, there has been increasing interest in CNCs as a promising key component to design new materials exhibiting optical functionality or mechanical high-performance, in context of the CNC's fascinations such as the nanoscale dimension with a high aspect ratio and the inherent high stiffness (Habibi et al. 2010; Klemm et al. 2011; Moon et al. 2011). For instance, CNCs can be ideal fillers for high-performance polymer nanocomposites, and, in fact, there have been many reports on the excellent filler effects of CNCs reinforcing various polymer matrices (Dufresne 2010; Eichhorn 2011; Miao and Hamad 2013).

In conditioning of the optical, mechanical, or thermo-mechanical properties of CNC-containing materials, the orientation control of CNCs is of great importance. Various attempts have been made to align CNCs in aqueous media and fix the orientation state in the dried films or polymer composites, for the purpose of making their properties anisotropic and upgraded. According to the situations, different methods were adopted to align CNCs; for example, CNCs were aligned in the suspensions by deformation of shearing (Nishiyama et al. 
1997) or drawing (Ureña-Benavides et al. 2010; Uddin et al. 2011; Gindl and Keckes 2007), and also by a non-contact technique of applying magnetic fields (Kvien and Oksman 2007; Pullawan et al. 2012; Tatsumi et al. 2014).

In the authors' preceding paper, we successfully demonstrated the investment of a clear mechanical anisotropy in polymer composites with CNC (Tatsumi et al. 2014); where the orientation control of CNC fillers was made using a magnetic alignment system. Briefly, the composites were prepared from cotton CNC/aqueous 2-hydroxyethyl methacrylate (HEMA) suspensions, via magnetic field application to the suspensions followed by polymerization of the monomer HEMA in the solvent. Applying a magnetic field $(8 \mathrm{~T})$ of static (s) or rotational (r) type to each mesophase of the phase-separated CNC suspensions resulted in fabrication of a composite of CNC/poly(2-hydroxyethyl methacrylate) (PHEMA) designated PHEMA-CNC $\mathrm{aniso}^{-\mathrm{S}}$ or PHEMA-CNC $\mathrm{aniso}^{-\mathrm{r}}$ A uniform structure of chiral nematic

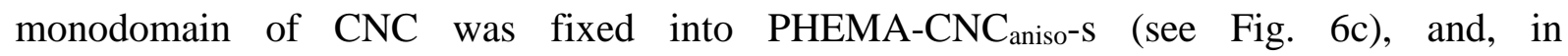
PHEMA-CNC $\mathrm{aniso}_{\mathrm{r}} \mathrm{r}$, CNC particles were distributed with their respective longer axes aligned normal to the plane of the field rotation (Fig. 6d).

In the present paper, we turned attention to a mechanical way to make a highly oriented CNC film from suspension under shear flow; it is just the way proposed by Nishiyama et al. (1997). They used a CNC sample isolated from the cell wall of a green alga, the particle size being $\sim 40 \mathrm{~nm}$ in diameter and $\sim 4 \mu \mathrm{m}$ in length (aspect ratio $\approx 100$ ). An aqueous suspension of the sulfuric acid-treated CNC was prepared at $1 \%$ in a glass vial, and this vial was kept horizontal and rotated around its center at $500 \mathrm{rpm}$. After $12 \mathrm{~h}$ rotation, a gel-like layer formed on the inner surface of the container was washed and dried into a solid film of CNC. The CNCs in the film were highly oriented in the direction of the applied shear flow. Consulting this Nishiyama's method, we prepared dry films of cotton-derived CNC from the aqueous suspensions by a similar shearing method, to see the alignment state of CNC attainable therein. Special care was exercised to $\mathrm{pH}$ condition of the starting suspension to 
be sheared. The obtained CNC films were characterized by optical and electron microscopy observations and wide-angle X-ray diffraction (WAXD) measurements. On the pattern and degree of CNC orientation, a comparative survey was made between the CNC films and the composites of PHEMA-CNC ${ }_{a n i s o-s}$ and PHEMA-CNC aniso-r.

\section{Experimental}

\section{Preparation of CNC}

The procedure for preparing the CNC used in this work was essentially the same as that described previously (Tatsumi et al. 2012). In brief, CNC particles were isolated from cotton cellulose powder (Whatman, CF11) by acid hydrolysis with 65 wt \% sulfuric acid, with mechanical stirring at $70{ }^{\circ} \mathrm{C}$ for $15 \mathrm{~min}$. After a dilution and centrifugation process, the fluid dispersion of CNC was dialyzed in distilled water for an adequate time period, and concentrated appropriately. The particle dimensions of CNC are $95 \mathrm{~nm}$ in length and $9 \mathrm{~nm}$ in diameter on average; the axial ratio is around 10. Surface sulfur content of the CNC particles (well refined) approximated $~ 1.1 \mathrm{wt} \%$, when determined by alkali titration.

\section{Preparation of CNC films by shearing method}

A concentrated $\mathrm{CNC} /$ water suspension $(\mathrm{CNC}=\sim 25 \mathrm{wt} \%)$ mentioned above was diluted and homogenized, and a suspension sample of $4 \mathrm{wt} \% \mathrm{CNC}$ (Suspension A) was prepared at $\mathrm{pH}=$ 2.0. Subsequently, half of this suspension was separately neutralized with addition of a small quantity of sodium hydroxide aqueous solution, and a $3.4 \mathrm{wt} \%$ CNC suspension (Suspension N) was prepared at $\mathrm{pH}=6.7$. They were about the isotropic-biphasic critical concentration, but used in the non-separating mixture before the phase separation. The suspensions of $4.9 \mathrm{~mL}$ (the former A) and $5.4 \mathrm{~mL}$ (the latter $\mathrm{N}$ ) were each put into a $20 \mathrm{~mL}$ glass vial of $25 \mathrm{~mm}$ in inner diameter. As illustrated in Figure 1, the vial was kept horizontal, 
and rotated around its center at $700 \mathrm{rpm}$ in an air blower of $40^{\circ} \mathrm{C}$; the tangential direction of the rotation is taken to correspond to the shear direction (SD). The shear rate applied was roughly $\sim 580 \mathrm{~s}^{-1}$ and $\sim 530 \mathrm{~s}^{-1}$ for Suspensions $\mathrm{A}$ and $\mathrm{N}$, respectively, by estimating the thickness $(1.5-1.7 \mathrm{~mm})$ of the respective fluids spread over the inside wall at an earlier stage of the rotation. About $20 \mathrm{~h}$ later, a dry film of CNC was formed on the inner surface of the glass wall. The film samples thus obtained were encoded by the $\mathrm{pH}$ value of the starting suspension: CNC-2.0 and CNC-6.7 from Suspensions A and N, respectively.

\section{$<<$ Figure $1>>$}

Substantially the same preparation of CNC films was repeated to confirm reproducibility of the orientation patterns observed for CNC-2.0 and CNC-6.7 by WAXD measurements, etc.

\section{Measurements}

Optical characterization of CNC films was made under a polarized optical microscope (POM), Olympus BX60F5. The film specimens were also observed by using a field emission scanning electron microscope (FE-SEM), Hitachi S-4800; they were sputter-coated with Pt before the observation.

WAXD measurements were made using a MAC Science Dip 2000 diffractometer equipped with an MXP18HF22 rotating anode generator. The measuring conditions were as follows: voltage and current of operation, $45 \mathrm{kV}$ and $84 \mathrm{~mA}$; X-ray wavelength, $0.154 \mathrm{~nm}$ (Ni-filtered $\mathrm{CuK} \alpha$ ); collimator size, $0.90 \mathrm{~mm}$; camera distance, $150 \mathrm{~mm}$; exposure time, $1800 \mathrm{~s} . \quad \mathrm{X}$-ray beam was irradiated perpendicular to the surface of the films.

\section{Results and discussion}

\section{Microscopic observations of CNC films}

Generally translucent films of CNC were obtained by the shearing method in either of the $\mathrm{pH}$ 
conditions, as the appearance is exemplified in Figure 2a. The CNC films were removed from the vial as several pieces, each showing a habit of curving along SD in some degree. The film thickness varied with location, e.g., from 0.1 to $0.5 \mathrm{~mm}$ in CNC-2.0, and the average value approximated $0.2 \mathrm{~mm}$ for CNC-2.0 and $0.35 \mathrm{~mm}$ for CNC-6.7; thicker parts of the films were, more or less, glossy and brownish. The inclination from the horizontal of the rotation axis in the process of shearing would be partly responsible for the variation and irregularity in film thickness. In the following microscopic and X-ray measurements, film specimens of 0.2-0.25 mm thick were mainly used.

Figure 2b and c show POM photographs of CNC-2.0 and CNC-6.7 samples, respectively; both photographs were taken by setting each sample nearly at an orthogonal position (SD $\| \boldsymbol{P}$ or $\boldsymbol{A}$ ) on the stage. The field of view was then considerably dark as a whole, when compared with the bright field in a diagonal setting, but the perfect extinction scarcely took place. Rather, as seen in both data, interference colors were still discernible and mingled with fine dark lines (more readily visible in Fig. 2b). This observation may be interpreted as being due to the essentially laminated fashion of the films, which were processed via repeated shear deformations of CNC suspensions while drying. The privileged optical axis of the respective oriented films was generally perpendicular to SD (CNC-2.0) and parallel to SD (CNC-6.7).

\section{$<<$ Figure $2>>$}

Selected data of FE-SEM observations for CNC films are given in Figure 3. In the observations, we found numerous fibrous entities (assimilation of CNCs) aligned mostly in good order in both CNC-2.0 and CNC-6.7 films. While the CNC alignment was preferentially parallel to SD in CNC-6.7 (Fig. 3c), the opposite, transverse alignment prevailed in CNC-2.0 (Fig. 3a). In the CNC-2.0 film, there also appeared a somewhat different fashion of fibrils' alignment in places; they formed a V- or U-like trajectory of orientation, as shown in Figure 3b. This morphology is apparently like the banded structure 
characteristic of shear-deformed polymer liquid crystals (Navard 1987; Nishio et al. 1985), but, rather, it may be regarded as the trace of a helical arrangement of CNCs (Majoinen et al. 2012; Tatsumi et al. 2012); viz., the chiral nematic structure could develop in the process of condensation of the sheared suspension and partly remain in the dry sample, as discussed below. Another attention should be paid to the fact that no trace of such a mesomorphic organization was observed in the CNC-6.7 film.

\section{$<<$ Figure $3>>$}

\section{CNC orientation distribution revealed by WAXD}

Figure 4a and b illustrate WAXD photographic data taken for films of CNC-2.0 and CNC-6.7, respectively; SDs of the two samples were both set horizontal, namely, parallel to the equator of the respective WAXD diagrams. The diffraction profiles of both data were characterized by cellulose I $\beta$ of a monoclinic crystal form (Sugiyama et al. 1991). This is instantiated by observation of the (200), (110), and (110) diffractions at $2 \theta=22.6^{\circ}, 16.6^{\circ}$, and $14.6^{\circ}$, respectively, each appearing as twined reflection arcs across the equator (Fig. 4a) or the meridian (Fig. 4b). Figure 5 displays the azimuthal scanning of the (200) diffraction intensity for the two samples used in Figure 4. In Figure 5a for CNC-2.0, we find two large peaks centered at azimuthal angles of $0^{\circ}$ and $180^{\circ}$; this definitely indicates that the $c$-axis of CNC and therefore the longitudinal axis tends to be aligned perpendicular to SD of the film. In Figure 5b for CNC-6.7, two peaks are located with the intensity maximum at azimuthal angles of $90^{\circ}$ and $270^{\circ}$; hence, the longer axes of CNCs are aligned preferentially parallel to SD of the film. These WAXD results correspond to the FE-SEM observations of fibrillar alignment that well distinguished between the two CNC films.

$<<$ Figure $4>>$

$<<$ Figure $5>>$ 
There were several investigations into shear-induced orientation phenomena of CNCs in the aqueous suspensions put into a rotating cylinder (Nishiyama et al. 1997; Orts et al. 1998; Ebeling et al. 1999); the suspensions were all acidic, and, except for the Nishiyama's case, the CNC concentrations were constant during shear flow in the respective samples and mostly above the critical concentration for ordered mesophase formation (at rest). Ebeling et al. (1999) reported a two-step process on the shear alignment of cotton-derived CNCs (aspect ratio $\approx 10)$ in a $6.9 \%(\mathrm{w} / \mathrm{v})$ aqueous suspension. That is, at lower shear rates $\left(<5 \mathrm{~s}^{-1}\right)$, CNCs were aligned nearly at random in SD but showed a preferential orientation in the vertical direction (in the plane perpendicular to SD), whereas, at higher shear rates of $\geq 5 \mathrm{~s}^{-1}$, CNCs were aligned individually parallel to SD. The estimation was based on small-angle X-ray scattering data obtained in two measuring setups, i) X-ray incidence being perpendicular to the surface of the sheared sample (normal view) and ii) that parallel to the flow direction SD (parallel or tangential view). The authors explained the result by assuming that CNCs formed anisotropic domains (i.e. mesophases) of uniplanar organization; the planar domains would orient along SD (each plane $\|$ SD) with the constituent CNCs distributed at random within the domains at low shear rates, before disruption at higher shear rates enabling the alignment of CNCs parallel to SD.

In the present work, the shear rates applied to CNC suspensions were $>500 \mathrm{~s}^{-1}$ beyond $5 \mathrm{~s}^{-1}$ in any case of preparing CNC-2.0 and CNC-6.7 films. The $c$-axis orientation of CNCs to SD observed for CNC-6.7 is qualitatively consistent with the results of high-shear experiments reported by Ebeling et al. (1999) and also by Orts et al. (1998); the latter group examined shear alignment of black spruce CNCs (aspect ratio, ca. 30-45) at suspension concentrations of 5-7 wt \%. With regard to CNC-2.0, however, the alignment state of CNCs is completely different than the common pattern despite the application of a similar high-rate shear (580 $\left.\mathrm{s}^{-1}\right)$, the longer axes of CNCs pointing to the transverse direction (TD) of the sheared sample. Accordingly, it is strongly suggested that the ionic atmosphere (which varies the critical CNC 
concentration of mesomorphic assembling) in the condensing suspensions would influence the CNC orientation in the films obtained under shear and drying.

A possible explanation for the present result is given in what follows. There will be two key points. One is basically correlated with the difference in ionic strength between Suspensions A and N. Suspension N (pH = 6.7) was prepared by neutralizing CNCs in the suspension equivalent to $\mathrm{A}(\mathrm{pH}=2.0)$ with $\mathrm{NaOH}$ aqueous solution. Therefore the counterions of the charged sulfate groups $\left(\mathrm{OSO}_{3}{ }^{-}\right)$on the surface of the CNCs in Suspension $\mathrm{N}$ are $\mathrm{Na}^{+}$, being changed from $\mathrm{H}^{+}$that should be the counterions on the CNCs in Suspension A. Dong et al. (1996; 1997) studied effects of ionic strength and counterions on the isotropic-chiral nematic phase transition of CNC suspensions by using various electrolytes as 227 additives. The studies demonstrated that well-refined CNC suspensions only with $\mathrm{H}^{+}$ counterions inherently associated with the surface sulfate groups formed an ordered phase at the lowest concentrations of the used crystallites, and the added electrolytes increased the critical concentration of isotropic-mesophase transition in any employment of acidic, neutral, or basic electrolytes. Accordingly, Suspension A would undergo the transition to form a 232 chiral nematic phase at an appreciably lower concentration than Suspension N, in the process 233 of water evaporation. This is supported by the SEM result (Fig. 3); the traces of chiral 234 nematic-like organization were surely observed for the CNC-2.0 film. Then, the nematic layers emerging in Suspension A under drying should be deformed in the high-shear flow of $>500 \mathrm{~s}^{-1}$, differing from the situation at much lower shear rates in the Ebeling and coworkers' 237 experiment (1999). In the present case using a rotated vessel, supposedly, the nematic layers 238 might have wound and even rolled up so that the directors were oriented perpendicular rather 239 than parallel to SD, as illustrated in Figure 6a. In shear flow of pulp suspensions, similar 240 behavior of rolling up or turning over of laterally assembled fibers has been experienced as a 241 flocculation process (Chen et al. 2003). By way of such a conversion of the arising ordered 242 phase from a planar nematic into rolled form (resulting in a transversely extended body), the 
sheared suspension A would eventually transform into a solid film of CNC-2.0, in which the majority of CNCs are aligned in TD while the minor traces of chiral nematic arrangement still remain. In the other neutralized suspension N, most probably, this CNC system with sodium counterions undergoes the transition to mesophase at higher concentration; instead, the individual nano-rods could readily align along the shear flow at an earlier stage of the rotation process, and the uniaxial orientation distribution of CNCs with reference to SD would continue until gelation of the colloidal system toward the dry film of CNC-6.7 (see Fig. 6b).

\section{$<<$ Figure $6>>$}

In relation to the orientation of CNCs perpendicular to SD, it is known in suspension rheology that, when neutrally buoyant spheroids or rigid rods (treated as non-Brownian particles) are subjected to shear flow in viscous fluid media, they conditionally drift into a 'log-rolling' state with the longer axis oriented in the vorticity direction (TD in the present paper) via a complicate kayaking motion; this is concisely summarized, for example, in an article by Gunes et al. (2008). Typically, the log-rolling phenomenon has been detected by rheo-optical techniques for dilute or semi-dilute suspensions of short fibers with dimensions of $>50 \mu \mathrm{m}$ diameter and $>1 \mathrm{~mm}$ length (Bartram et al. 1975; Iso et al. 1996). The applicability and contribution of such a log-rolling effect to the shear-induced orientation of CNC particles are undigested and unknown. The former orientation mechanism stated above is still hypothetical but offers a more convincing interpretation for the distinctive experimental result.

A serious question remains unresolved, however. It is the orientation pattern of CNC-6.7 (Fig. 6b), not of CNC-2.0 (Fig. 6a), that is in accordance with the earlier observation by Nishiyama et al. (1997) for a shear-deformed CNC film. There was no sign of the CNC orientation to TD in their study. A determinate factor in causing the result is the extremely high aspect ratio of the alga-derived CNC used there, the axial ratio being 10 times larger than that of cotton-derived CNC. Plainly the former particles are much more advantageous 
to attainment of the uniaxial alignment along the shear flow. This should result in the smooth development of CNC arrays oriented parallel to SD in the dry film.

\section{Comparison of orientation development between various samples}

To estimate the degree of CNC orientation for the present shear-deformed films, the orientation parameter $f\left(=\left(3<\cos ^{2} \phi_{c}>-1\right) / 2\right)$ of the $c$-axis of CNC was quantified. In a similar way to that adopted in our previous paper (Tatsumi et al. 2014), the calculation was done by using the relation $f=-2 f_{a}$ assuming a cylindrically symmetric distribution of CNC rods around TD (CNC-2.0) or SD (CNC-6.7); where $f_{a}$ is the orientation parameter of the $a$-axis, which can be determined from the azimuthal data of the (200) diffraction intensity (Fig. 5). As a consequence, we obtained $f=0.56$ for CNC-2.0 and $f=0.68$ for CNC-6.7 (see upper two rows in Table 1), each data being an average with \pm 0.1 tolerance for three specimens belonging to the same sheared sample. The higher degree of orientation development in CNC-6.7 than in CNC-2.0 was in consistency with an FE-SEM observation indicating the distinction in orderliness of fibrillar alignment between the two samples.

$<<$ Table $1>>$

As regards the case of CNC-6.7, the orientation parameter of $\sim 0.7$ is comparable with the one reported by Ebeling et al. (1999) for a cotton-derived CNC suspension (6.9 \%, see above); they determined $f \approx 0.75$ by small-angle $\mathrm{X}$-ray scattering for the fluid under shear at a rate of $1700 \mathrm{~s}^{-1}$. Meanwhile, Orts et al. (1998) observed higher values of 0.8 or 0.9 as the order parameter of black spruce CNCs (axial ratio of 33 or 47) in the aqueous suspensions (5 or 7 wt \% CNC) sheared at rates of $\geq 1000 \mathrm{~s}^{-1}$, where the measurement was made by use of a small-angle neutron scattering technique. However, these data (ranging from $\sim 0.7$ to 0.9 ) of the orientation parameter are all lower than $f=0.96$ obtained by Nishiyama et al. (1997) for the shear-oriented film of green alga-derived CNC; probably, the primacy in orientation development of the latter would come from the extraordinarily high axial ratio of CNC, $\sim 100$. 
Figure 6 also includes schematic illustrations of the orientation patterns of CNCs observed previously for PHEMA-CNC composites synthesized via magnetic field application to mesomorphic CNC suspensions (Tatsumi et al. 2014); two products were termed

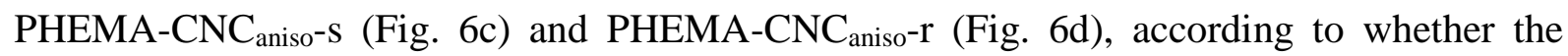
magnetic field $(\boldsymbol{B})$ was static (s) or rotating (r). As shown in Table 1, we have obtained $f=$ 0.84 (as to uniaxial alignment of the chiral nematic helical axis) for PHEMA-CNC aniso-s and $f$ $=0.60$ (as to uniaxial alignment of the longer axis of CNC) for PHEMA-CNC ${ }_{\text {aniso-r; }}$ these are in a parallel or somewhat higher level, when compared with the data of $f \approx 0.6-0.7$ for the shear-oriented CNC films using the same cotton cellulose. For any of the four samples, however, a passable level of orientation achievement may be admitted from a practical standpoint regarding the design of CNC-core polymer materials showing definite optical or mechanical anisotropy. The shearing method makes CNCs align to a certain degree with simpler equipment and procedure, while the non-contact technique using magnetic fields excels at aligning the fillers highly and uniformly.

\section{Conclusions}

Oriented films of cotton-derived CNC (axial ratio, $\sim 10$ ) were prepared from aqueous suspensions under high-rate shear $\left(>500 \mathrm{~s}^{-1}\right)$ accompanied by water evaporation. The film products were named CNC-2.0 and CNC-6.7 according as the starting suspension was in an acidic $(\mathrm{pH}=2.0)$ or neutral $(\mathrm{pH}=6.7)$ condition; the neutral suspension $(\mathrm{N})$ was made by addition of $\mathrm{NaOH}$ into the parent suspension (A) of $\mathrm{pH}=2.0$. Comparative characterization of the oriented CNC films was performed by POM and FE-SEM microscopy and WAXD measurements.

The films were optically birefringent and composed of fine fibrillar structures aligned mostly in good order; the running way of the fibrous entities was, in perspective, parallel to 
321 the shear direction (SD) in CNC-6.7, but perpendicular to SD in CNC-2.0. In correspondence with the microscopic observations, WAXD analysis revealed that the longitudinal axes of CNCs were aligned preferentially perpendicular to SD in CNC-2.0 and parallel to SD in CNC-6.7. In the drying process of the sheared suspensions A and N, probably, the formation of (chiral) nematic ordered phase occurred in A, but scarcely did in $\mathrm{N}$, which stands on the difference in the counterions of surface-sulfated CNCs between the two $\left(\mathrm{H}^{+}\right.$in $\mathrm{A}$ and $\mathrm{Na}^{+}$in $\left.\mathrm{N}\right)$. This deduction was also supported by FE-SEM observations of surface mophology for the corresponding two dry films. In the suspension $\mathrm{N}$ free of mesomorphic structuring, therefore, CNC particles were allowed to individually align parallel to SD so as to produce the common uniaxial orientation in the CNC-6.7 film. With regard to the suspension A under a rotatory shear flow, it was assumed that the nematic planar domains arriving with time were deformed by winding-up force so that the nematic directors were arranged perpendicular to SD, which gave rise to the transverse orientation pattern of the elemental CNC rods in the CNC-2.0 film.

The degree of orientation development $(f \approx 0.6-0.7)$ in the two CNC films was taken to be in a parallel or somewhat lower level, in comparison with the orientation achievement of the

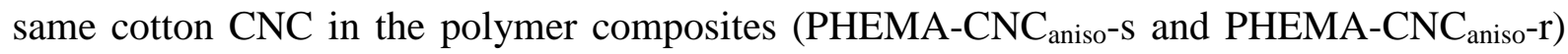
that were previously synthesized under a static or rotational magnetic field. However, the success in controlling the CNC orientation with the ionic condition of the sheared suspension is of great significance, from both scientific and practical points of view.

Acknowledgments This work was financially supported by a Grant-in-Aid for Scientific Research (A) (No. 26252025 to Y.N.) from the Japan Society for the Promotion of Science.

\section{Compliance with Ethical Standards}

The authors declare no conflict of interest. 


\section{References}

Bartram E, Goldsmith HL, Mason SG (1975) Particle motions in non-Newtonian media III. further observations in elasticoviscous fluids. Rheol Acta 14:776-782

Chen B, Tatsumi D, Matsumoto T (2003) Fiber orientation and flow properties of pulp fiber suspensions under shear flow conditions. Sen'i Gakkaishi 59:471-478

Dong XM, Kimura T, Revol J-F, Gray DG (1996) Effects of ionic strength on the isotropic-chiral nematic phase transition of suspensions of cellulose crystallites. Langmuir 12:2076-2082

Dong XM, Gray DG (1997) Effects of counterions on ordered phse formation in suspensions of charged rodlike cellulose crystallites. Langmuir 13:2404-2409

Dong XM, Revol J-F, Gray DG (1998) Effect of microcrystallite preparation conditions on the formation of colloid crystals of cellulose. Cellulose 5:19-32

Dufresne A (2010) Processing of polymer nanocomposites reinforced with polysaccharide nanocrystals. Molecules 15:4111-4128

Ebeling T, Paillet R, Borsali R, Diat O, Dufresne A, Cavaillé J-Y, Chanzy H (1999) Shear-induced orientation phenomena in suspensions of cellulose microcrystals, revealed by small angle X-ray scattering. Langmuir 15:6123-6126

Eichhorn SJ (2011) Cellulose nanowhiskers: promising materials for advanced applications. Soft Matter 7:303-315

Gray DG (1994) Chiral nematic ordering of polysaccharides. Carbohydr Polym 25:277-284

Gindle W, Keckes J (2007) Drawing of self-reinforced cellulose films. J Appl Polym Sci 103:2703-2708

Gunes DZ, Scirocco R, Mewis J, Vermant J (2008) Flow-induced orientation of non-spherical particles: effect of aspect ratio and medium rheology. J Non-Newtonian Fluid Mech 
374

Habibi Y, Lucia LA, Rojas OJ (2010) Cellulose nanocrystals: chemistry, self-assembly, and 375 applications. Chem Rev 110:3479-3500

Iso Y, Koch DL, Cohen C (1996) Orientation in simple shear flow of semi-dilute fiber suspensions 1. weaky elastic fluids. J Non-Newtonian Fluid Mech 62:115-134

Klemm D, Kramer F, Moritz S, Lindström T, Ankerfors M, Gray DG, Dorris A (2011) Nanocelluloses: a new family of nature-based materials. Angew Chem Int Ed 50:5438-5466

Kvien I, Oksman K (2007) Orientation of cellulose nanowhiskers in polyvinyl alcohol. Appl Phys A: Mater Sci Process 87:641-643

Majoinen J, Kontturi E, Ikkala O, Gray DG (2012) SEM imaging of chiral nematic films cast from cellulose nanocrystal suspensions. Cellulose 19:1599-1605

Marchessault RH, Morehead FF, Walter NM (1959) Liquid crystal systems from fibrillar polysaccharides. Nature 184:632-633

Miao C, Hamad WY (2013) Cellulose reinforced polymer composites and nanocomposites: a critical review. Cellulose 20:2221-2262

Moon RJ, Martini A, Nairn J, Simonsen J, Youngblood J (2011) Cellulose nanomaterials review: structure, properties and nanocomposites. Chem Soc Rev 40:3941-3994

Navard P (1987) Optical properties of a shear-deformed thermotropic cellulose derivative. J Polym Sci Part B Polym Phys 25:1089-1098

Nishio Y, Yamane T, Takahashi T (1985) Morphological studies of liquid-crystalline cellulose derivatives. II. Hydroxypropyl cellulose films prepared from liquid-crystalline aqueous solutions. J Polym Sci Polym Phys Ed 23:1053-1064

Nishiyama Y, Kuga S, Wada M, Okano T (1997) Cellulose microcrystal film of high uniaxial orientation. Macromolecules 30:6395-6397

Orts WJ, Godbout L, Marchessault RH, Revol J-F (1998) Enhanced ordering of liquid 
399 crystalline suspensions of cellulose microfibrils: a small angle neutron scattering study.

$400 \quad$ Macromolecules 31:5717-5725

401 Pullawan T, Wilkinson AN, Eichhorn SJ (2012) Influence of magnetic field alignment of 402 cellulose whiskers on the mechanics of all-cellulose nanocomposites. Biomacromolecules $403 \quad 13: 2528-2536$

404 Revol J-F, Bradford H, Giasson J, Marchessault RH, Gray DG (1992) Helicoidal self-ordering 405 of cellulose microfibrils in aqueous suspension. Int J Biol Macromol 14:170-172 406 Sugiyama J, Vuong R, Chanzy H (1991) Electron diffraction study on the two crystalline 407 phases occurring in native cellulose from an algal cell wall. Macromolecules 24:4168-4175 408 Tatsumi M, Kimura F, Kimura T, Teramoto Y, Nishio Y (2014) Anisotropic polymer 409 composites synthesized by immobilizing cellulose nanocrystal suspensions specifically oriented under magnetic fields. Biomacromolecules 15:4579-4589

Tatsumi M, Teramoto Y, Nishio Y (2012) Polymer composites reinforced by locking-in a 412 liquid-crystalline assembly of cellulose nanocrystallites. Biomacromolecules 13:1584-1591 413 Uddin AJ, Araki J, Gotoh Y (2011) Toward "strong" green nanocomposites: polyvinyl 414 alcohol reinforced with extremely oriented cellulose whiskers. Biomacromolecules $415 \quad 12: 617-624$

416 Ureña-Benavides EE, Brown PJ, Kitchens CL (2010) Effect of jet stretch and particle load on 417 cellulose nanocrystal-alginate nanocomposite fibers. Langmuir 26:14263-14270 
Figure Captions

420

Figure 1. Schematic illustration of preparation of oriented CNC films from aqueous suspension by shearing under evaporation. The sample container (glass vial) was rotated at 700 rpm.

Figure 2. Visual appearance of a strip of CNC-2.0 (panel a), and polarized optical micrographs of CNC-2.0 (panel b) and CNC-6.7 (panel c) printed with a scale bar of $50 \mu \mathrm{m}$. White arrows indicate the shear direction (SD) of films.

Figure 3. FE-SEM images of shear-deformed CNC films: (a) and (b) data for CNC-2.0; (c) data for CNC-6.7. White arrows indicate SD, and black arrows guide the alignment direction of fibrous entities (assimilation of CNCs) to the eye.

Figure 4. WAXD patterns of shear-deformed CNC films: (a) data for CNC-2.0; (b) data for

CNC-6.7. White arrows indicate SD of the film specimens.

Figure 5. Azimuthal intensity scans of the (200) diffraction of CNCs: (a) data for CNC-2.0;

(b) data for CNC-6.7. These two specimens are the same as those used in Figure 4.

Figure 6. Different orientation patterns of CNC rods observed for shear-deformed CNC films, (a) CNC-2.0 and (b) CNC-6.7, and polymer composites, (c) PHEMA-CNCaniso-s and

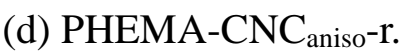

443 In addition to the six figures, there is one table (Table 1). 
444 Table 1. Evaluations of orientation parameters of the longitudinal axis of CNC for dry CNC

445 films obtained under shear in different $\mathrm{pH}$ conditions and for PHEMA-CNC $\mathrm{Caniso}_{\text {composites }}$

446 synthesized under different magnetic fields

\begin{tabular}{ccc}
\hline Sample & Orientation parameter $(f)$ & Reference axis of sample $^{\mathrm{b}}$ \\
\hline CNC-2.0 & 0.56 (avg.) & // TD \\
CNC-6.7 & 0.68 (avg.) & $/ /$ SD \\
\hline PHEMA-CNC aniso-S $_{\text {PHEMA-CNC }}$ aniso-r & 0.84 (helix axis) ${ }^{\mathrm{a}} ;-0.42\left(\mathrm{CNC}\right.$ axis) ${ }^{\mathrm{a}}$ & $/ / \boldsymbol{B}$ \\
PHE & $0.60^{\mathrm{a}}$ & $/ / \boldsymbol{N}$ \\
\hline
\end{tabular}

${ }^{a}$ Quoted from a previous paper (Tatsumi et al. 2014)

b See schematic illustrations in Fig. 6 
450

451

452

453

454

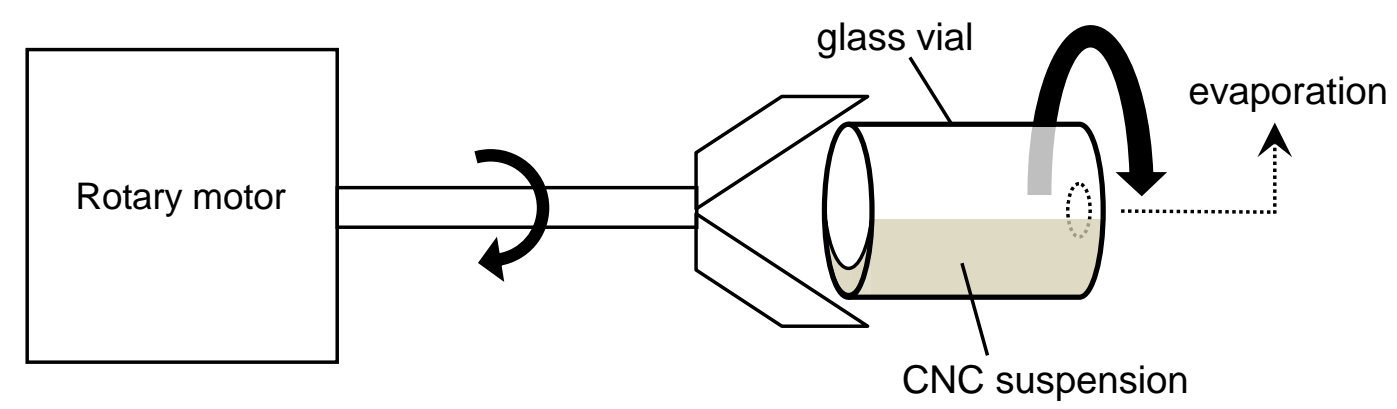

455

456 Figure 1. Schematic illustration of preparation of oriented CNC films from aqueous 457 suspension by shearing under evaporation. The sample container (glass vial) was rotated at $458700 \mathrm{rpm}$.

459

460 

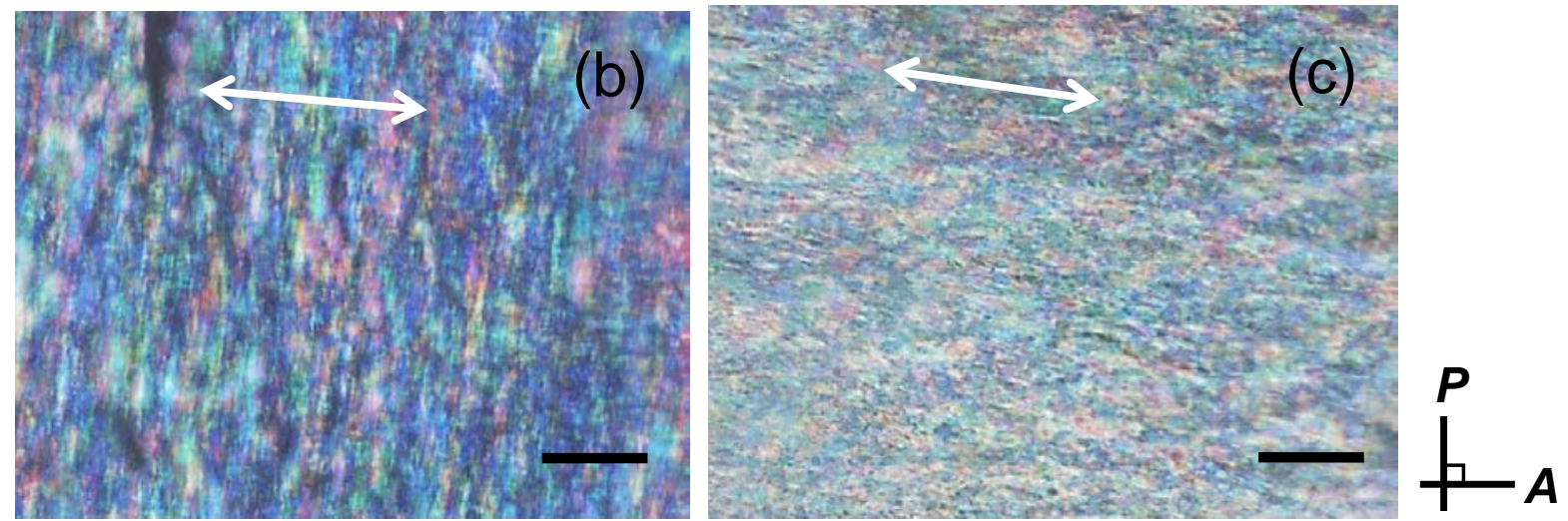

463

464 Figure 2. Visual appearance of a strip of CNC-2.0 (panel a), and polarized optical 465 micrographs of CNC-2.0 (panel b) and CNC-6.7 (panel c) printed with a scale bar of $50 \mu \mathrm{m}$. 466 White arrows indicate the shear direction (SD) of films. 

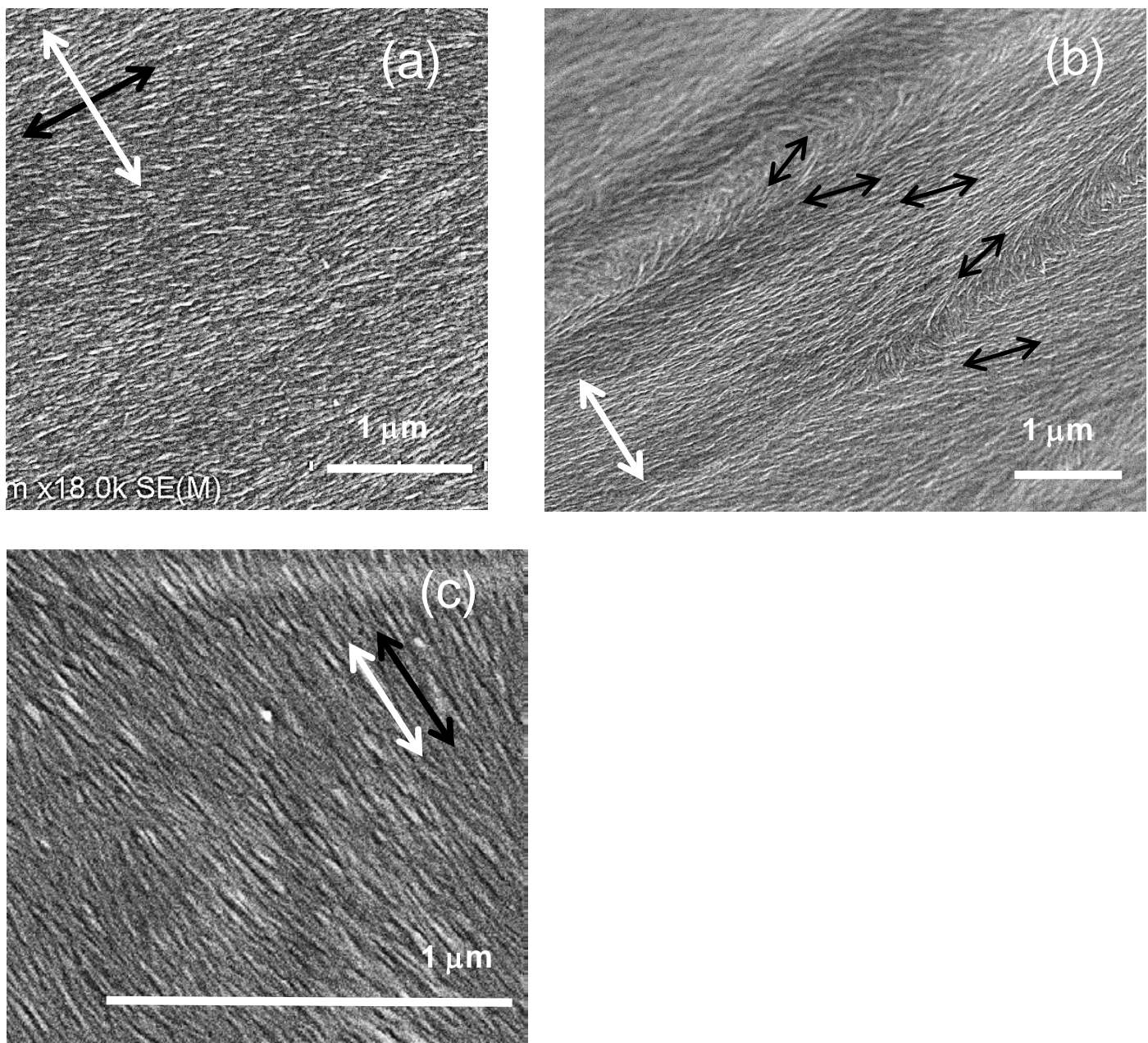

470

471 Figure 3. FE-SEM images of shear-deformed CNC films: (a) and (b) data for CNC-2.0; (c)

472 data for CNC-6.7. White arrows indicate SD, and black arrows guide the alignment

473 direction of fibrous entities (assimilation of CNCs) to the eye. 

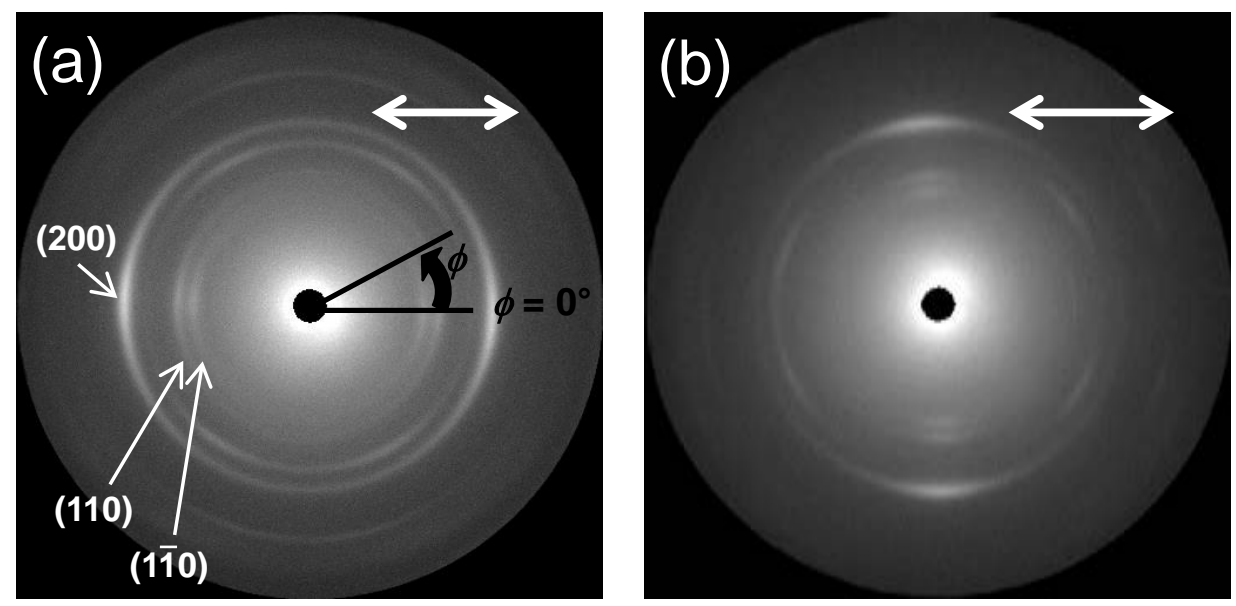

476

477 Figure 4. WAXD patterns of shear-deformed CNC films: (a) data for CNC-2.0; (b) data for 478 CNC-6.7. White arrows indicate SD of the film specimens.

479

480 

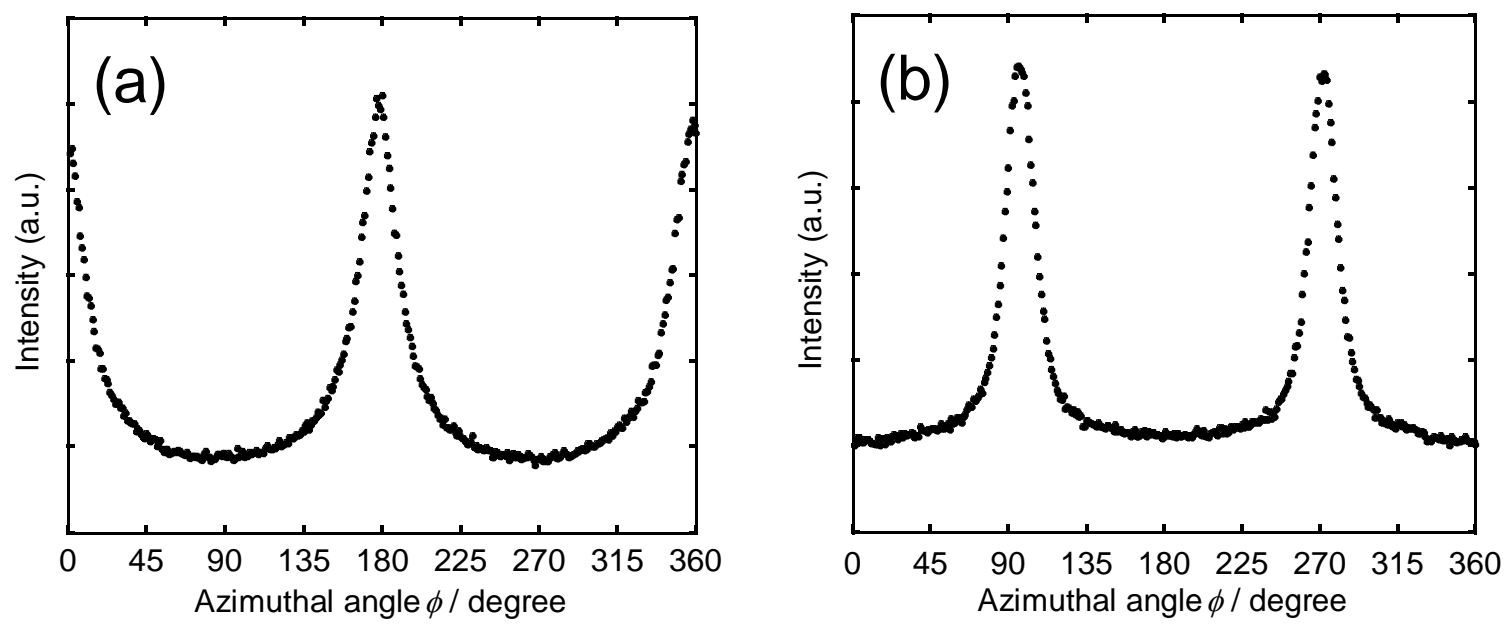

482

483 Figure 5. Azimuthal intensity scans of the (200) diffraction of CNCs: (a) data for CNC-2.0;

484 (b) data for CNC-6.7. These two specimens are the same as those used in Figure 4. 
(a)

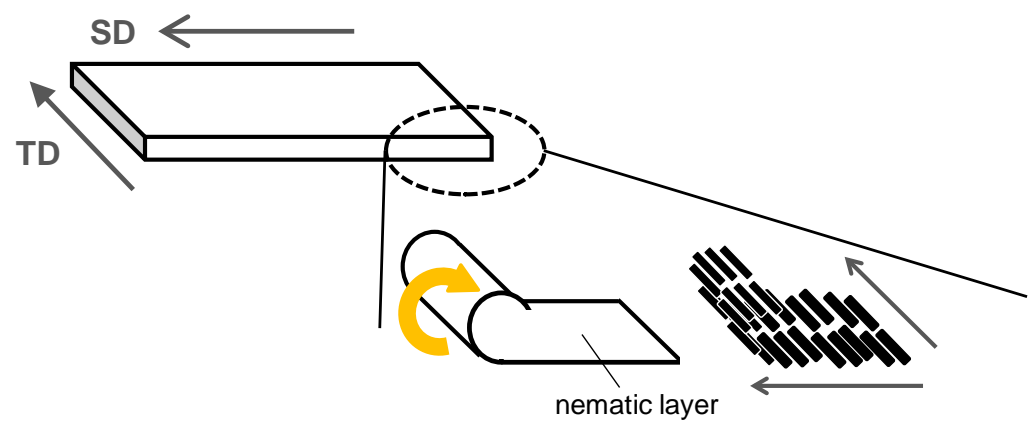

(b)

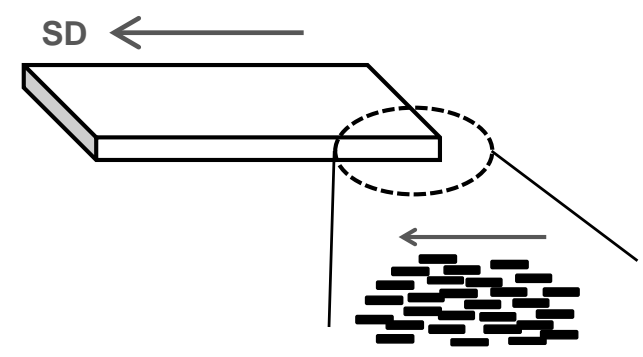

(c)

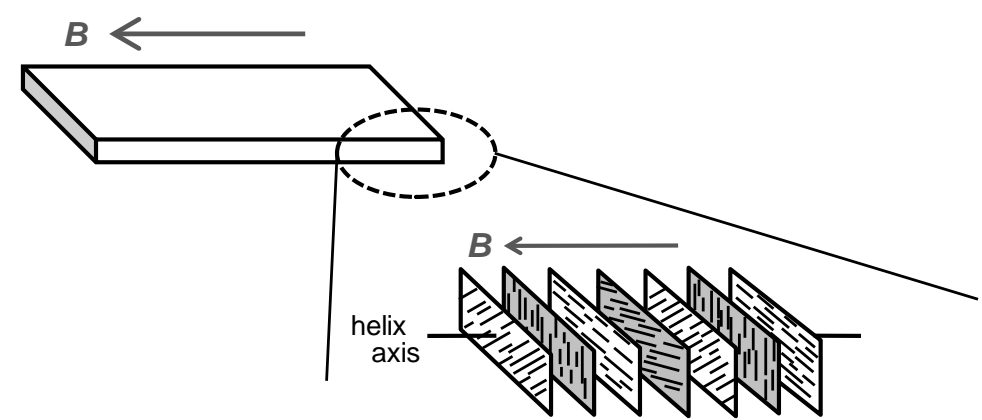

(d)

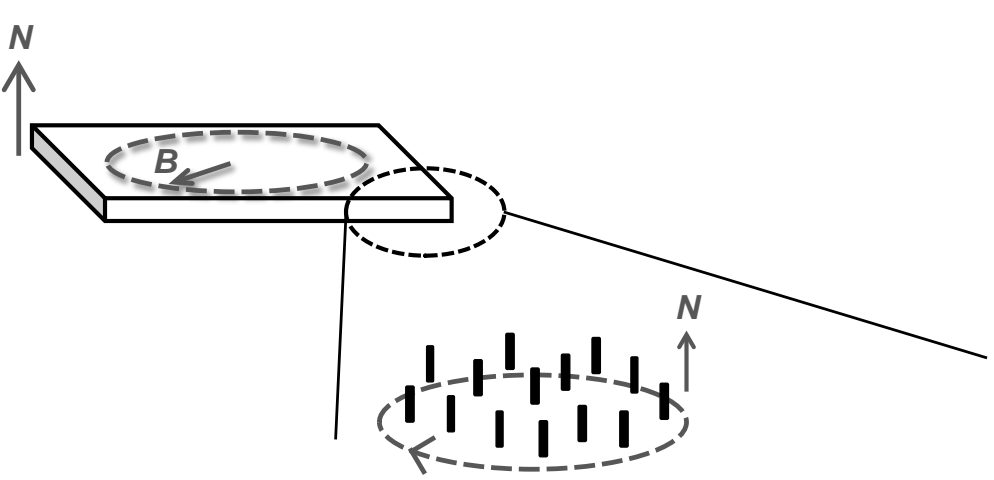

Figure 6. Different orientation patterns of CNC rods observed for shear-deformed CNC

493 films, (a) CNC-2.0 and (b) CNC-6.7, and polymer composites, (c) PHEMA-CNCaniso-s and

(d) PHEMA-CNC aniso-r. 